\title{
Modified mini-maze via left thoracic cavity under VATS for paroxysmal atrial fibrillation in a patient with complete interruption of the inferior vena cava
}

\author{
Fan $\mathrm{He}^{1}$, Bijun $\mathrm{Xu}^{1}$, Shiqiang Wang ${ }^{1}$, Huai-Dong Chen ${ }^{2}$, and Weimin Zhang ${ }^{1}$ \\ ${ }^{1}$ Zhejiang University School of Medicine Sir Run Run Shaw Hospital \\ ${ }^{2}$ Sir Run Run Shaw Hospital, School of Medicine, Zhejiang University
}

January 16, 2021

\begin{abstract}
Objectives: We sought to determine the technical feasibility of surgical bipolar radiofrequency ablation (endoscopic maze procedure) through the left chest cavity in patients with an interrupted inferior vena cava (IVC). Methods and Results: A 57year-old female with paroxysmal atrial fibrillation (AF) and an interrupted IVC was referred to our hospital for radiofrequency ablation. Transseptal puncture and left atrium (LA) ablation failed through a standard IVC approach via the femoral vein due to intrahepatic interruption of IVC. We performed a modified surgical bipolar radiofrequency ablation (RF) on the beating heart through 3 ports in the left chest wall. Pulmonary vein isolation and ablation of the left atrium were achieved by bipolar radiofrequency ablation. Ganglionic plexus ablation was completed using the ablation pen. The left atrial appendage was excluded. No complications occurred during or after the procedure. The patient was discharged with sinus rhythm 3 days later after the procedure. She was taking amiodarone (100mg bid) within 6 months after the procedure, and had no recurrence of AF. Conclusions: We successfully performed a modified mini-maze procedure in a patient with paroxysmal AF and IVC interruption through the left thoracic cavity under video-assisted thoracoscopic surgery (VATS). We can successfully complete pulmonary vein (PV) isolation, left atrium box isolation, cardiac ganglia ablation, Marshall ligament ablation, and coronary sinus epicardium ablation using this technique.
\end{abstract}

\section{Introduction}

The duplication of the inferior vena cava (IVC) is a rare anatomical variation occurs during embryological development of the IVC ${ }^{[1]}$. This anomaly interrupts the IVC, which renders the ablation of atrial fibrillation (AF) impossible with the standard approach via IVC to left atrium. To address the technical difficulty, we adopted the surgical bipolar radiofrequency ablation (endoscopic maze procedure) through the left chest cavity in patients with an interrupted IVC.

\section{Case Report}

A 58-year-old female patient was presented with recurrent palpitation for one month. Holter electrocardiography monitoring showed paroxysmal AF. She did not respond to oral anti-arrhythmia medicine and was admitted for catheter ablation. Echocardiography revealed a $40 \mathrm{~mm}$ left atrium in anteroposterior diameter and $62 \%$ left ventricular ejection fraction (LVEF). During the procedure, cardiologist could not put the catheter into the right atrium. Angiography showed interruption of the IVC. Further computed tomographic venography (CTV) showed double IVC with intrahepatic interruption, azygos and hemiazygos vein continuation, and intrahepatic venous shunt. The hepatic vein was shown to connect the right atrium (Figure $1)$. 
We decided to perform Modified mini-maze via left thoracic cavity under VATS on this patient. The procedure was performed on the beating heart through 3 ports in the left chest wall (Figure 2). The pulmonary vein isolation and ablation of the left atrium were achieved by bipolar radiofrequency ablation with AtriCure Isolator Synergy ablation clamp(Figure 3). Ganglionic plexus ablation was completed using the AtriCure Isolator Synergy ablation pen. The left atrial appendage was excluded. The operation was very successful and no complications occurred during or after the procedure. The patient was discharged with sinus rhythm 3 days later after the procedure. She was taking amiodarone (100mg bid) within 6 months after the procedure, and had no recurrence of $\mathrm{AF}$.

\section{Discussion and Conclusion}

Congenital IVC malformation reflects the complicated multi-segmental development of the IVC during embryogenesis ${ }^{[2]}$. It is classified by Huntington and McLure with up to 14 theoretical variations ${ }^{[3]}$. The most common anomalies of the IVC include the circumaortic left renal vein (1.5\%-8.7\%), the retroaortic left renal vein $(2.1 \%)$, the double IVC $(0.2 \%-3 \%)$, azygos, the hemi-azygos continuation of IVC $(0.6 \%)$, and the isolated left-sided IVC $(0.2 \%-0.5 \%)^{[4]}$. The Double IVC, the azygos and hemiazygos continuation, the intrahepatic IVC interruption, and the transhepatic venous shunt were rare. Here we reported the patient with the intrahepatic shunt on the right and hemi-azygos system on the left for the venous return from extremities. The patient had the retroaortic anastomotic vein connecting 2 venous collateral pathways towards the heart. Most patients with IVC malformation are asymptomatic. The prevalence of thromboembolic disease in patients with a duplicated IVC is unknown, yet there are case reports of pulmonary embolism in those patients ${ }^{[4]}$.

Cardiologist may use a superior vena cava (SVC) approach for transseptal puncture through the right internal jugular vein in LA ablation, but that is not a conventional choice. It is impossible to puncture the atrial septal by IVC approach in patients with IVC anomaly. The Cox Maze III procedure has been considered the "gold standard" to eliminate AF, but it is not well accepted by patients because of its invasiveness. Wolf and colleagues introduced using the thoracoscopic technique to perform Cox Maze procedure ${ }^{[5]}$. However, the procedures are technically challenging. It was further modified, such as Mei who reported a complete thoracoscopic ablation of the LA via the left chest to treat lone atrial fibrillation ${ }^{[6]}$. The ablation circuit was shown in figure 4. We used Mei's method on the beating heart through 3 ports in the left chest wall. Pulmonary vein isolation and ablation of the left atrium were achieved by bipolar radiofrequency ablation. Ganglionic plexus ablation was completed using the ablation pen. The left atrial appendage was excluded. By using this modified procedure, we shortened the operation time, simplified workload of anesthetists, and reduced injury for patients by avoiding thoracotomy, minimizing changes of patient's position during the surgery, and simplifying the trachea cannula management by the anesthetists. In conclusion, we have shown that the modified Cox Maze III procedure under VATS has technical advantage and feasible especially to AF patients with IVC anomalies.

The sinus rhythm was restored in the patient after surgery. She has been well followed up for 2 years since the operation with no recurrence of AF. The results indicate that the modified Cox Maze III procedure is efficient, safe, convenient and reproducible for treatment of paroxysmal or persistent atrial fibrillation. More cases and further follow-up data should be collected to substantiate our findings.

\section{Author contributions}

Conceptualization: Shiqiang Wang. Data analysis: Huaidong Chen. Writing - original draft: Fan He. Writing - review \& editing: Bijun Xu. Approval of article: Weimin Zhang. Funding secured by Fan He.

\section{Reference}

1. Giordano JM, Trout HH III. Anomalies of the inferior vena cava. J Vasc Surg. 1986;3(6):924-928.

2. McClure CFW, Butler EG. The development of the vena cava inferior in man. Am J Anat. 1925;35(3):331-383. 
3. Maxwell EW, Erwin GS. Four cases of anomalous inferior vena cava with an explanation of their developmental origin. J Anat. 1928;62(pt 2):184-197.

4. Sahin H, Pekcevik Y, Aslaner R. Double Inferior Vena Cava (IVC) With Intrahepatic Interruption, Hemiazygos Vein Continuation, and Intrahepatic Venous Shunt. Vasc Endovascular Surg. 2017 Jan;51(1):38-42. doi:10.1177/1538574416687734. PubMed PMID: 28100158.

5. Wolf RK, Schneeberger EW, Osterday R, Miller D, Merrill W, Flege JB Jr, et al. Video-assisted bilateral pulmonary vein isolation and left atrial appendage exclu- sion for atrial fibrillation. $\mathrm{J}$ Thorac Cardiovasc Surg. 2005;130:797-802.

6. Mei J, Ma N, Ding F, Chen Y, Jiang Z, Hu F, Xiao H. Complete thoracoscopic ablation of the left atrium via the left chest for treatment of lone atrial fibrillation. J Thorac Cardiovasc Surg. 2014 Jan;147(1):242-6.doi:10.1016/j.jtcvs.2012.10.005. Epub 2012 Nov 2. PubMed PMID: 23122696.

\section{Legends}

Figure 1 The CTV showed double IVC with intrahepatic interruption, azygos and hemiazygos vein continuation, and intrahepatic venous shunt. The hepatic vein was shown to connect the right atrium.

Figure 2 The procedure was performed through 3 ports in the left chest wall. The camera port $(10 \mathrm{~mm})$ was introduced in the eighth intercostal space at the subscapular angle line. The first working port (25

$\mathrm{mm}$ ) was introduced about $30 \mathrm{~mm}$ anterior to the subscapular angle line in the sixth intercostal space. The second working port $(10 \mathrm{~mm})$ was introduced $30 \mathrm{~mm}$ posterior to the subscapular angle line in the seventh intercostal space.

Figure3. The right and left pulmonary vein isolation were achieved by bipolar radiofrequency ablation device.

Figure 4. The linear lesion from the left inferior pulmonary vein to the right superior pulmonary vein by the bipolar radiofrequency ablation clamp. PV, Pulmonary vein; SVC, superior vena cava; IVC, inferior vena cava.

\section{Hosted file}

figures.pdf available at https://authorea.com/users/389806/articles/504344-modified-minimaze-via-left-thoracic-cavity-under-vats-for-paroxysmal-atrial-fibrillation-in-a-

patient-with-complete-interruption-of-the-inferior-vena-cava 